\title{
Optical Beam Characterisation via Phase-Space Tomography
}

\section{Caracterización de Haces Ópticos vía Tomografía del Espacio de Fases}

\author{
A. Cámaras \\ Depto. Óptica, Universidad Complutense de Madrid. Avda. Complutense S/N 48040 Madrid, Spain. \\ ${ }^{(*)}$ E-mail: a.camara.iglesias@gmail.com $\quad$ S: SEDOPTICA member
}

DOI: $10.7149 /$ OPA.49.4.49502

\begin{abstract}
:
This work presents four methods based on phase-space tomography for the characterization of the spatial structure of optical beams - the results of my $\mathrm{PhD}$ thesis. Each technique is optimal for characterizing beams with a particular symmetry. All methods are experimentally exercised to prove their feasibility in real world applications.
\end{abstract}

Key words: Phase-space tomography, beam characterization, coherence retrieval

\section{RESUMEN:}

Este trabajo presenta cuatro métodos basados en la tomografía en el espacio de fases para la caracterización de la estructura espacial de haces ópticos: los resultados de mi tesis doctoral. Cada técnica está optimizada para caracterizar haces con una simetría en concreto. Todos los métodos son probados experimentalmente, demostrando así su utilidad para aplicaciones del mundo real.

Palabras clave: Tomografía espacio de fases, caracterización de haces, reconstrucción de coherencia

\section{REFERENCES AND LINKS / REFERENCIAS Y ENLACES}

[1] J. A. Rodrigo, T. Alieva, "Illumination coherence engineering and quantitative phase imaging," Opt. Lett. 19, 5634-5637 (2014). https://doi.org/10.1364/OL.39.005634

[2] F. Dubois, L. Joannes, J.-C. Legros, "Improved three-dimensional imaging with a digital holography miscroscope with a source of partially spatial coherence," Appl. Opt. 38, 7085-7094 (1999). https://doi.org/10.1364/A0.38.007085

[3] J. C. Ricklin, F. M. Davidson, "Atmospheric optical communication with a Gaussian Schell beam," J. Opt. Soc. Am. A, 20, 856-866 (2003). https://doi.org/10.1364/JOSAA.20.000856

[4] M. Santarsiero, R. Borghi, "Measuring spatial coherence by using a reversed-wavefront Young interferometer," Opt. Lett. 31, 861-863 (2006). https://doi.org/10.1364/OL.31.000861

[5] A. I. González, Y. Mejía, "Nonredundant array of apertures to measure the spatial coherence in two dimensions with only one interferogram," J. Opt. Soc. Am. A 28, 1107-1113 (2011). https://doi.org/10.1364/JOSAA.28.001107

[6] D. Mendlovic, G. Shabtay, A. W. Lohman, N. Konforti, "Display of spatial coherence," Opt. Lett. 23, 1084-1086 (1996). https://doi.org/10.1364/OL.23.001084

[7] D. Dragoman, "Can the Wigner transform of a two-dimensional rotationally symmetric beam be fully recovered from the Wigner transform of its one-dimensional approximation?," Opt. Lett 25, 281-283 (2000).

https://doi.org/10.1364/OL.25.000281 
[8] L. Waller, G. Situ, J. W. Fleischer, "Phase-space measurement and coherence synthesis of optical beams," Nature Photon. 6, 474-479 (2012). https://doi.org/10.1038/nphoton.2012.144

[9] L. Tian, J. Lee, S. B. Oh, G. Barbastathis, "Experimental compressive phase space tomography," Opt. Express 20, 8296-8308 (2012). https://doi.org/10.1364/OE.20.008296

[10] M. G. Raymer, M. Beck, D. F. McAlister, "Complex wave-field reconstruction using phase-space tomography," Phys. Rev. Lett. 72, 1137-1140 (1994). https://doi.org/10.1103/PhysRevLett.72.1137.

[11] A. Cámara. Optical beam characterisation via phase-space tomography, Tesis Doctoral, Univ. Complutense de Madrid. Dir. M. L. Calvo y T. Alieva, Septiembre (2014). http://eprints.ucm.es/27244/1/T35439.pdf

[12] A. Cámara, Optical Beam Characterization via Phase-Space Tomography. Springer (2015). https://doi.org/10.1007/978-3-319-19980-1

[13] T. Ditmire, E. T. Gumbrell, R. A. Smith, J.W. G. Tisch, D. D. Meyerhofer, M. H. R. Hutchinson, "Spatial coherence measurement of soft X-Ray radiation produced by high order harmonic generation," Phys. Rev. Lett. 77, 4756-4759 (1996). https://doi.org/10.1103/PhysRevLett.77.4756

[14] A. Cámara, T. Alieva, J. A. Rodrigo, M. L. Calvo, "Phase-space tomography with a programmable Radon-Wigner display," Opt. Lett. 36, 2441-2443 (2011). https://doi.org/10.1364/OL.36.002441

[15] A. Cámara, T. Alieva, J. A. Rodrigo, M. L. Calvo, "Phase space tomography reconstruction of the Wigner distribution for optical beam separable in Cartesian coordinates," J. Opt. Soc. Am. A 26, 1301-1306 (2009). https://doi.org/10.1364/JOSAA.26.001301

[16] A. Cámara, T. Alieva, I. Castro, J. A. Rodrigo, "Phase space tomography for characterization of rotationally symmetric beams," J. Opt. 16, 015705 (2014). https://doi.org/10.1088/2040-8978/16/1/015705

[17] A. Cámara, J. A. Rodrigo, T. Alieva, "Optical coherenscopy based on phase-space tomography," Opt. Express 21, 13169-13183 (2013). https://doi.org/10.1364/OE.21.013169

[18] J. A. Rodrigo, T. Alieva, M. L. Calvo, "Experimental implementation of the gyrator transform," J. Opt. Soc. Am. A 24, 3135-3139 (2007). https://doi.org/10.1364/JOSAA.24.003135

\section{Introducción}

El estado de coherencia de un haz óptico juega papel primordial en la formación de imagen. Como se ha demostrado, por ejemplo en [1], las franjas de difracción de un objeto dependen, no solo de su forma, pero también del estado de coherencia del haz con el que se le ilumina. Aunque utilizar luz parcialmente coherente mejora los resultados de muchas aplicaciones originalmente propuestas para luz coherente, como microscopía cuantitativa, holografía digital [2] o comunicaciones ópticas en el espacio libre [3,4], este tipo de haces no es utilizado comúnmente. El mayor impedimento a su aplicación a gran escala es la carencia de métodos que permitan caracterizar en un tiempo reducido el estado de coherencia de un haz, es decir, la recuperación de su intensidad mutua (MI). Varias técnicas interferométricas y difractivas han sido propuestas para determinar experimentalmente la MI [4,.5]. Algunas de las técnicas han triunfado para casos particulares, como haces que satisfacen ciertas simetrías [6,7] o al hacer asunciones sobre el modelo de coherencia del haz [8,9]. Sin embargo, la mayor dificultad consiste en el desarrollo de una técnica adecuada para aplicaciones del mundo real que permita caracterizar haces bidimensionales parcialmente coherentes con estructura espacial arbitraria.

En lugar de definir un haz por su MI, es posible utilizar descripciones equivalentes como la distribución de Wigner (WD) o la función de ambigüedad (AF). La ventaja de estas funciones alternativas es que pueden ser directamente recuperadas experimentalmente aplicando la tomografía en el espacio de fases (phase space tomography en inglés, PST) [10]. La PST requiere el registro de una cantidad significativa de medidas de intensidad y su posterior procesado numérico. Su utilidad ha sido demostrada anteriormente para situaciones simplificadas: para caracterizar haces unidimensionales [6], haces invariantes respecto a 
rotación [7], haces cercanos a la incoherencia [8] o compuestos por unos pocos modos incoherentes [9]. Sin embargo los beneficios de la PST para la caracterización de haces bidimensionales con estructura espacial arbitraria están sin explotar.

La tesis [11] resumida en este trabajo propone varias técnicas originales basadas en la PST para la caracterización del estado de coherencia de haces. Esto incluye un procedimiento novedoso para recuperar la MI de haces bidimensionales que es demostrado experimentalmente en varios ejemplos involucrando luz con estado de coherencia parcial. También se proponen y demuestran experimentalmente otras técnicas para caracterizar haces con ciertas simetrías: haces unidimensionales, haces separables en coordenadas cartesianas y haces rotacionalmente simétricos. Todos los métodos son agnósticos del estado de coherencia de los haces e incluyen un procedimiento de verificación para comprobar que el haz a caracterizar posee las simetrías requeridas por la técnica. Todos los métodos se demuestran experimentalmente utilizando sistemas ópticos diseñados o modificados para dicho propósito. Los principales resultados resumidos aquí han sido publicados en [12].

\section{Tomografía en el Espacio de Fase}

Un haz de luz linealmente polarizado y cuasimonocromático se puede definir en un plano transversal a su dirección de propagación por una función escalar $f(\mathbf{r})$, de las coordenadas del plano $\mathbf{r}=(x, y)$, llamada amplitud del campo. Cuando el haz es parcialmente coherente, $f$ es una función estocástica producida por un gran número de contribuciones aleatorias, por lo que $f$ sigue una estadística gaussiana. Esto implica que el haz queda completamente definido por sus momentos estadísticos de segundo orden, es decir, su intensidad mutual (MI):

$$
\Gamma\left(\mathbf{r}_{1}, \mathbf{r}_{2}\right)=\left\langle f\left(\mathbf{r}_{1}\right) f^{*}\left(\mathbf{r}_{2}\right)\right\rangle
$$

donde $\langle\cdot\rangle$ representa el promedio respecto a múltiple realizaciones del haz y $*$ representa el complejo conjugado. Toda la información del haz se puede extraer de la MI. La intensidad se define como, $I=\Gamma(\mathbf{r}, \mathbf{r})$, mientras que el grado de coherencia se define como, $\gamma\left(\mathbf{r}_{1}, \mathbf{r}_{2}\right)=\Gamma\left(\mathbf{r}_{1}, \mathbf{r}_{2}\right) / \sqrt{I\left(\mathbf{r}_{1}\right) I\left(\mathbf{r}_{2}\right)}$.

Recuperar la MI de un haz arbitrario a partir de medidas de intensidad es una tarea complicada y tediosa. Afortunadamente existen representaciones alternativas para haces parcialmente coherentes que, como demostraremos más adelante, se pueden reconstruir experimentalmente con mayor facilidad que la MI. Las representaciones más utilizadas son la WD [12]:

$$
W(\mathbf{r}, \mathbf{p})=\frac{1}{\sigma^{2}} \int \mathrm{d} \mathbf{r}^{\prime} \Gamma\left(\mathbf{r}+\mathbf{r}^{\prime} / 2, \mathbf{r}-\mathbf{r}^{\prime} / 2\right) \exp \left(-\frac{\mathrm{i} 2 \pi}{\sigma^{2}} \mathbf{p} \cdot \mathbf{r}^{\prime}\right)
$$

y la $A F$ :

$$
A(\mathbf{r}, \mathbf{p})=\frac{1}{\sigma^{2}} \int \mathrm{d} \mathbf{r}^{\prime} \Gamma\left(\mathbf{r}^{\prime}+\mathbf{r} / 2, \mathbf{r}^{\prime}-\mathbf{r} / 2\right) \exp \left(-\frac{\mathrm{i} 2 \pi}{\sigma^{2}} \mathbf{p} \cdot \mathbf{r}^{\prime}\right)
$$

Como se aprecia por sus definiciones, tanto la WD como la AF son funciones que involucran una coordenada espacial, $\mathbf{r}$, y otra en el espacio conjugado (de Fourier), $\mathbf{p}=(u, v)$. Es por esto que estas funciones se dicen representaciones en el espacio de fases. Nótese que para recuperar la intensidad del haz original se debe proyectar la función WD sobre el espacio $\mathbf{r}$ :

$$
I(\mathbf{r})=\frac{1}{\sigma^{2}} \int \mathrm{d} \mathbf{p} W(\mathbf{r}, \mathbf{p})=\frac{1}{\sigma^{2}} \int \mathrm{d} \mathbf{p} A(2 \mathbf{r}, \mathbf{p})
$$

Este resultado será útil más adelante para establecer la PST.

Cuando la luz se propaga por un sistema óptico, se produce una evolución de la amplitud de campo, y por tanto de la MI, WD y AF. En concreto, para sistemas ópticos lineales no absorbentes definidos por la matriz $4 \times 4$ de transformación $\mathrm{ABCD}, \mathbf{T}=[\mathbf{A}, \mathbf{B} ; \mathbf{C}, \mathbf{D}]$, el efecto en la WD y la AF viene dado por las siguientes transformaciones afines [12]:

$$
W(\mathbf{r}, \mathbf{p})=W_{\mathbf{T}}(\mathbf{A r}+\mathbf{B p}, \mathbf{C r}+\mathbf{D} \mathbf{p}), A(\mathbf{r}, \mathbf{p})=A_{\mathbf{T}}(\mathbf{A r}+\mathbf{B p}, \mathbf{C r}+\mathbf{D} \mathbf{p}),
$$

donde $t$ representa la operación de transposición.

La tomografía en el espacio de fases es una técnica de reconstrucción de la WD o AF de un haz que explota la Eq. (5) para el caso concreto de sistemas que producen la transformada fraccionaria de Fourier (FRFT). En notación $A B C D$, la FRFT se define como: 


$$
\mathbf{A}=\mathbf{D}=\left[\begin{array}{cc}
\cos \gamma_{x} & 0 \\
0 & \cos \gamma_{y}
\end{array}\right], y \mathbf{B}=-\mathbf{C}=\left[\begin{array}{cc}
\sin \gamma_{x} & 0 \\
0 & \sin \gamma_{y}
\end{array}\right]
$$

Introduciendo el anterior resultado en la Eq. (5), es fácil comprobar que la FRFT es una operación que produce la rotación en espacio de fases de la WD y de la AF de un haz parametrizada por los ángulos $\gamma_{x}$ y $\gamma_{y}$. Por tanto, siguiendo Eq. (4), la intensidad de un haz después de atravesar un sistema FRFT viene dada por:

$$
S^{\gamma_{x}, \gamma_{y}}(\mathbf{r})=\frac{1}{\sigma^{2}} \int \mathrm{d} \mathbf{p} W\left(\left[\begin{array}{cc}
\cos \gamma_{x} & 0 \\
0 & \cos \gamma_{y}
\end{array}\right] \mathbf{r}+\left[\begin{array}{cc}
\sin \gamma_{x} & 0 \\
0 & \sin \gamma_{y}
\end{array}\right] \mathbf{p},\left[\begin{array}{cc}
\cos \gamma_{x} & 0 \\
0 & \cos \gamma_{y}
\end{array}\right] \mathbf{p}-\left[\begin{array}{cc}
\sin \gamma_{x} & 0 \\
0 & \sin \gamma_{y}
\end{array}\right] \mathbf{r}\right),
$$

lo que se corresponde a la proyección de la WD o la AF en un plano del espacio de fases definido por los parámetros de la transformación. Esta medida de intensidad, $S^{\gamma_{x}, \gamma y}(\mathbf{r})$, se conoce como espectro fraccionario de Fourier de potencia.

La tomografía consiste en la reconstrucción de la función (objeto) a partir de sus proyecciones usando un conjunto de proyecciones, $S^{\gamma_{x}, \gamma_{y}}(\mathbf{r})$, que cubren homogéneamente todas las direcciones del espacio de fases. Se puede recuperar la WD original (la MI o la AF) utilizando un algoritmo de reconstrucción tomográfíca como filtered backprojection. Por lo tanto, el método PST consiste en los siguientes pasos:

1. Medir un conjunto de espectros fraccionarios de Fourier para ángulos independientes y equidistantes en un rango $\pi / 2$.

2. Reconstruir la WD o la AF a través de la doble inversión tomográfica de las medidas obtenidas: primero para las variables $(x, u)$ y después para $(y, v)$.

Aunque la PST es un método relativamente sencillo, implica una serie de operaciones que lo hacen poco eficiente y, en consecuencia, impráctico para un caso general. Por ejemplo, suponiendo que tomamos 32 proyecciones para cada valor de un ángulo de los espectros fraccionarios de potencia, eso conlleva la medida de $32 \times 32=1024$ espectros fraccionarios de potencia. Por otro lado, se necesitan todas las proyecciones para realizar la primera reconstrucción del punto 2 del método. Esta operación, que conlleva la transformación de una cantidad muy significativa de información, no está preparada para ser ejecutada en paralelo, ya que el resultado de la primera reconstrucción es necesario para empezar la segunda reconstrucción.

Estos problemas han impedido la aplicación de la PST en general excepto en casos muy concretos donde el método se ha podido simplificar considerablemente. A continuación expondremos los métodos que establece la tesis [11]. Tres de ellos son una simplificación de la PST posible gracias a simetrías del haz. El último método no asume ninguna simetría, pero produce información de forma incremental, es decir sin procesar toda la información de golpe, y además es susceptible a computación en paralelo.

\section{Radon-Wigner Display}

En ciertas situaciones no es posible, o deseable, caracterizar completamente la estructura espacial de un haz bidimensional, ya que asumiendo ciertas hipótesis el análisis de un perfil unidimensional es suficiente. Esto es así para el estudio de la coherencia espacial de haces de rayos X [13] y haces invariantes respecto a rotación, para los que una caracterización completa se puede obtener de la WD de uno de los perfiles radiales [7]. Por lo tanto, el análisis de la estructura espacial de haces unidimensionales es un problema relevante para multitud de aplicaciones.

Se pueden describir los haces unidimensionales por una amplitud de campo unidimensional, $f(x)$. Así, la $\mathrm{MI}, \Gamma\left(x_{1}, x_{2}\right)$, y la $\mathrm{WD}, W(x, u)$, son funciones bidimensionales. Esto simplifica significativamente la adquisición, almacenamiento de las proyecciones fraccionarias de Fourier para su procesado en la WD del haz. Además, existen sistemas ópticos, llamados Radon-Wigner display (RWD), que permiten medir todas las proyecciones de la WD de un haz unidimensional en una única medida de intensidad [6].

En la tesis presentada [11] se propone un sistema RWD para la caracterización de haces unidimensionales que utiliza dos moduladores espaciales de luz (SLMs). El esquema del sistema se presenta en la Fig. 1. Cada SLM se divide en canales horizontales. Cada canal se comporta como una lente de diferente focal de forma que el canal $i$ de cada SLM genera una modulación de fase dada por la expresión:

$$
t_{1}(x, j)=\exp \left[-\frac{\mathrm{i} \pi x^{2}}{s^{2}}\left(2-\frac{\cot \gamma_{j}}{2}\right)\right], t_{2}(x, j)=\exp \left[-\frac{\mathrm{i} 4 \pi x^{2}}{s^{2}}\left(1-\sin \gamma_{N-j * 1}\right)\right],
$$


que produce la FRFT para ángulo $\gamma_{j}$, donde $s=\sqrt{2 \lambda z}$ es el parámetro de escala del sistema que depende de la longitud de onda de la luz empleada, $\lambda$, y la distancia entre SLMs, $z$. Adicionalmente se introducen sendas lentes convergentes cilíndricas con modulación de fase en la dirección vertical $\mathrm{L}_{1} \mathrm{y} \mathrm{L}_{2}$ de focal $z$. En el plano de la cámara CCD se obtiene una intensidad que se corresponde con el espectro fracionario de Fourier de la señal, repetida en la dirección vertical, incidente en $\mathrm{SLM}_{1}$.

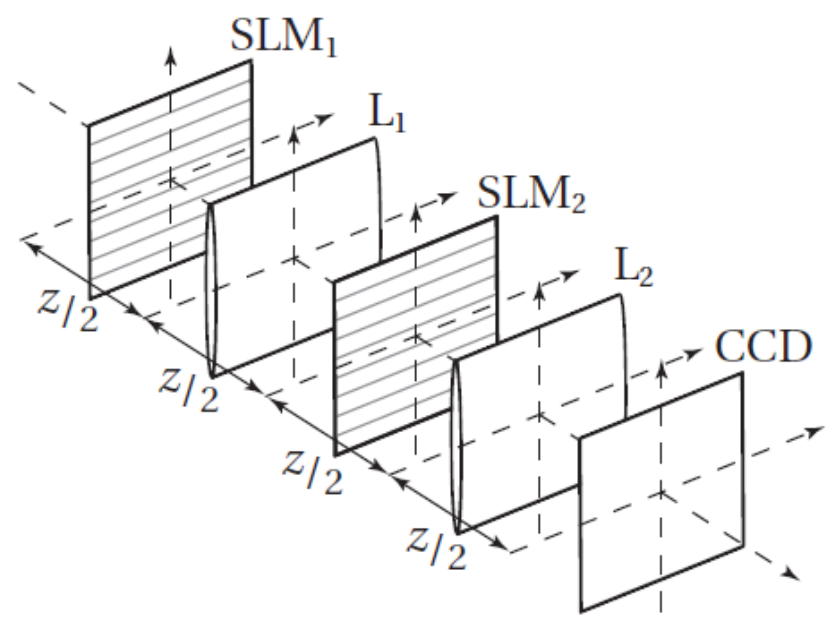

Fig. 1. Sistema óptico RWD para la caracterización de haces unidimensionales.

La incorporación del SLM mejora el rendimiento del sistema en comparación con el resto de sistemas ópticos que utilizan únicamente elementos analógicos. Al ser el SLM un elemento digital, se puede programar para producir diferentes rangos de proyecciones fraccionarias de Fourier en varias medidas. Dichas medidas se pueden juntar posteriormente para incrementar la precisión de la WD reconstruida. Experimentalmente se probó esta ventaja para un haz rectángulo con modulación cuadrática de fase [14]:

$$
f(x)=\operatorname{rect}\left(\frac{x}{w}\right) \exp \left(\frac{\mathrm{i} \pi x^{2}}{\lambda \mathrm{f}_{\mathrm{c}}}\right)
$$

siendo $w=2,47 \mathrm{~mm}$ la anchura del rectángulo y $\mathrm{f}_{\mathrm{c}}=0,93 \mathrm{~mm}$ el parámetro de chirp. De esa forma, el parámetro de chirp obtenido a partir de la WD reconstruida a partir de una única medida de 293 proyecciones (ver Fig. 2a y Fig. 2b) es menos preciso que el obtenido a partir de dos medidas unidas formando 586 proyecciones (ver Fig. 2c y Fig. 2d). La Tabla 1 resume los resultados obtenidos.

El sistema RWD propuesto en la tesis es una mejora con respecto del estado del arte, ya que su naturaleza programable le permite ser flexible con las medidas obtenidas.

TABLA 1. Resultados experimentales para el parámetro de chirp.

\begin{tabular}{|c|c|c|}
\hline \hline Situación & $\mathrm{f}_{\mathrm{c}}(\mathrm{mm})$ & $\Delta \mathrm{f}_{\mathrm{c}}(\mathrm{mm})$ \\
\hline \hline Única medida & 1.09 & 0.03 \\
Doble medida & 0.905 & 0.014 \\
Teórico & 0.93 & - \\
\hline \hline
\end{tabular}



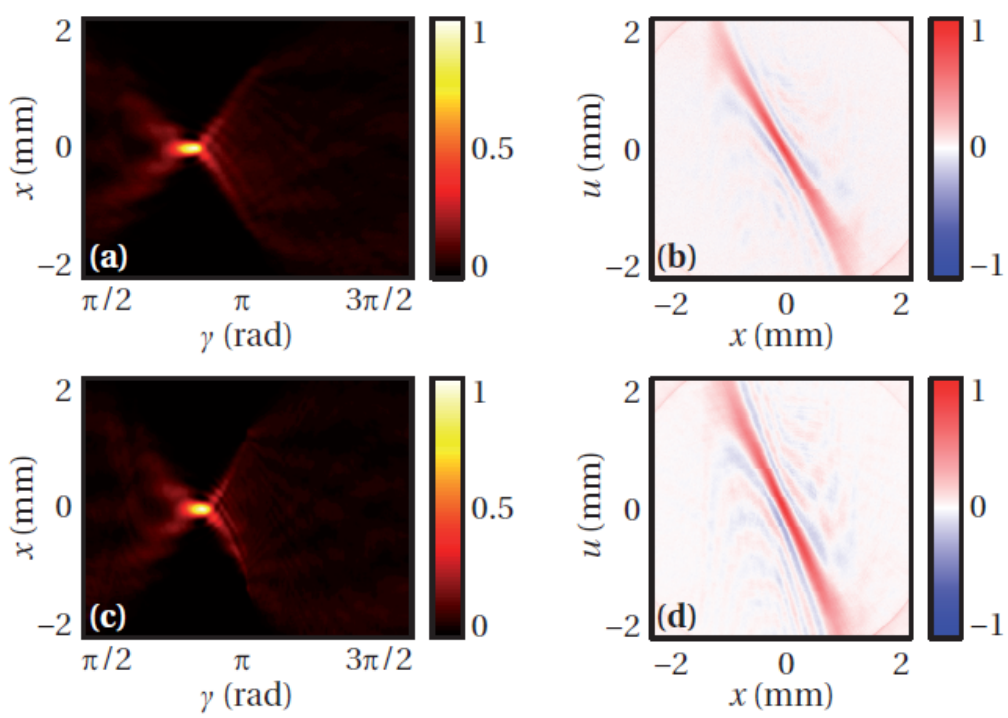

Fig. 2. Medida de intensidad (columna izquierda) y WD reconstruida (columna derecha) para una única medida conteniendo 293 proyecciones (fila superior) y dos medidas unidas conteniendo un total de 586 proyecciones (fila inferior).

\section{Haces Separables en Coordenadas Cartesianas}

Un haz es separable en coordenadas cartesianas si su MI se puede factorizar en funciones independientes que únicamente dependen de una coordenada espacial (y su variable espectral asociada):

$$
\Gamma\left(\mathbf{r}_{1}, \mathbf{r}_{2}\right)=\Gamma_{x}\left(x_{1}, x_{2}\right) \Gamma_{y}\left(y_{1}, y_{2}\right) .
$$

Este tipo de haces son comúnmente utilizados en experimentos, por ejemplo, los haces Hermite-Gauss (HG), que son modos fundamentales de láseres.

La caracterización de haces separables usando PST se puede simplificar sobremanera teniendo en cuenta que la propiedad de separabilidad se transmite a la WD y a los espectros fraccionarios de potencia:

$$
W(\mathbf{r}, \mathbf{p})=W_{x}(x, u) W_{y}(y, v), \quad \mathrm{S}^{\gamma_{x}, \gamma_{y}}(\mathbf{r})=\mathrm{S}^{\gamma_{x}}(x) \mathrm{S}^{\gamma_{y}}(y) .
$$

Nótese que los espectros fraccionarios unidimensionales para ángulos $\gamma_{x}, \gamma_{y}$ se pueden obtener proyectando en un eje cartesiano los espectros fraccionarios bidimensionales para ángulos $\gamma_{x}, \gamma_{y}$ :

$$
\int \mathrm{d} y S^{\gamma_{x}, \gamma_{y}}(\mathbf{r})=A_{y} S^{\gamma_{x}}(x), \quad \int \mathrm{d} x S^{\gamma_{x}, \gamma_{y}}(\mathbf{r})=A_{x} S^{\gamma_{y}}(y),
$$

donde $A_{x}$ y $A_{y}$ son constantes que no dependen del ángulo de la FRFT. Por lo tanto, la PST se puede formular para haces separables como sigue [10]:

1. Medir espectros fraccionarios para ángulos independientes $\gamma_{x}, \gamma_{y}$, ambos cubriendo un rango continuo de longitud $\pi / 2$. (Intervalo reducido por la simetría).

2. Integrar los espectros fraccionarios bidimensionales para obtener los espectros fraccionarios unidimensionales asociados a cada coordenada cartesiana.

3. Reconstruir las WD asociadas a cada coordenada cartesiana a partir de los espectros fraccionarios unidimensionales.

4. Reconstruir la WD del haz bidimensional multiplicando las WDs asociadas a cada coordenada cartesiana.

Esta técnica fue demostrada experimentalmente utilizando un haz HG [15]:

$$
f(\mathbf{r})=\mathrm{HG}_{4,3}(\mathbf{r}) \exp \left(-\frac{\mathrm{i} \pi \mathbf{r}^{2}}{\lambda \mathrm{f}_{\mathrm{c}}}\right),
$$

con longitud de onda, $\lambda=532 \mathrm{~nm}$, y parámetro de chirp, $\mathrm{f}_{\mathrm{c}}=2 \mathrm{~m}$. Para ello obtuvimos los espectros fraccionarios de Fourier bidimensionales asociados con la FRFT antisimétrica, es decir, $\gamma_{y}=-\gamma_{x}$, utilizando el sistema mostrado en Fig. 3a. 


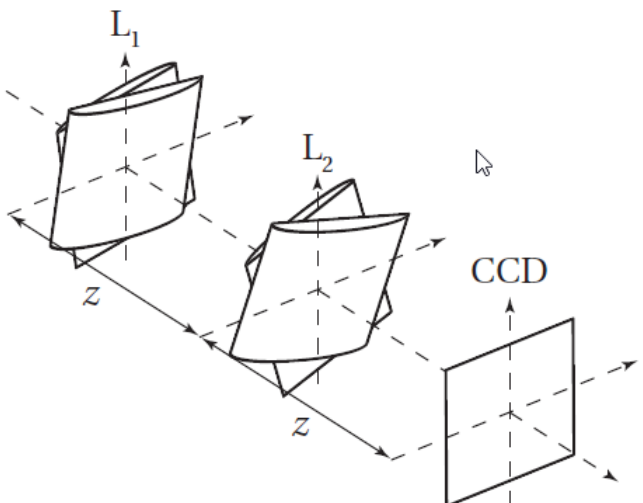

(a)

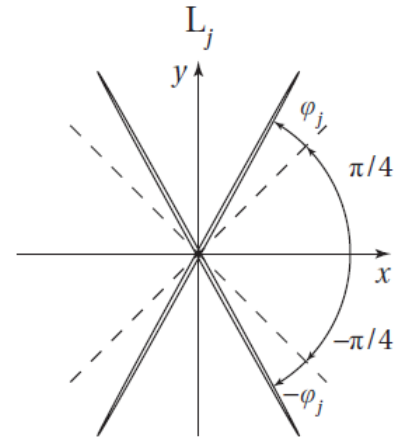

(b)

Fig. 3. (a) Esquema del sistema para obtener los espectros fraccionarios de potencia antisimétricos. (b) Relación entre ángulos de cada par de lentes cilíndricas.

El sistema óptico está formado por dos pares de lentes cilíndricas separadas una distancia $z$. Cada lente cilíndrica puede rotar alrededor del eje óptico del sistema. Rotaciones concretas de lentes producen la transformación fraccionaria de Fourier antisimétrica del haz de entrada. Consideremos $\theta_{i, j}$ el ángulo respecto al eje $X$ de la lente $i$ del par $j$, y definamos los ángulos auxiliares $\varphi_{j}$ :

$$
\theta_{j, 1}=\varphi_{j}+\pi / 4, \quad \theta_{j, 2}=-\varphi_{j}-\pi / 4 \text {. }
$$

En la Fig. 3b se muestra un esquema de la relación entre ángulos. El ángulo de la FRFT antisimétrica, $\gamma$, se relaciona con los ángulos $\varphi_{j}$ mediante esta expresión:

$$
\sin \left(2 \varphi_{1}\right)=\cot (\gamma / 2) / 2, \sin \left(2 \varphi_{2}\right)=\sin \gamma .
$$

En el experimento tomamos 45 proyecciones bidimensionales antisimétricas. A partir de ellas reconstruimos las WDs mostradas en la Fig. 4.

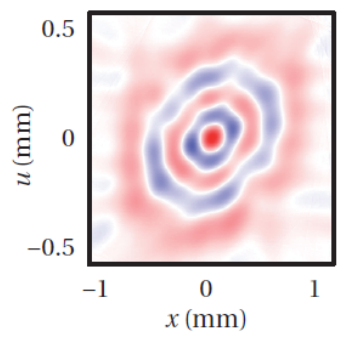

(a)

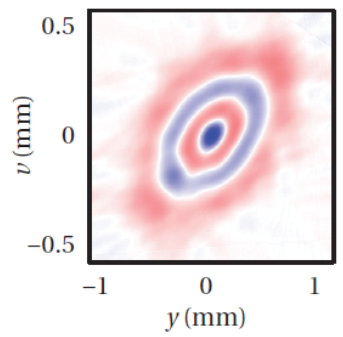

(c)

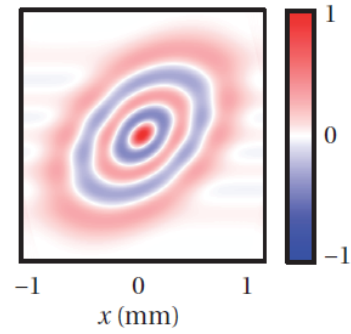

(b)

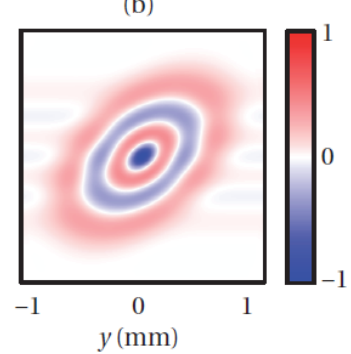

(d)

Fig. 4. WD experimental (columna izquierda) y teórica (columna derecha) asociadas con cada coordenada cartesiana.

Estos resultados demuestran la viabilidad del método para la caracterización de haces separables en coordenadas cartesianas utilizando un sistema óptico basado en lentes cilíndricas.

\section{Haces Rotacionalmente Simétricos}

Un haz es rotacionalmente simétrico si su MI cumple la siguiente condición para cualquier valor de theta:

$$
\Gamma\left(\mathbf{r}_{1}, \boldsymbol{r}_{2}\right)=\Gamma\left[\mathbf{M}(\theta) \mathbf{r}_{1}, \mathbf{M}(\theta) \mathbf{r}_{2}\right]
$$


donde

$$
\mathbf{M}(\theta)=\left[\begin{array}{cc}
\cos \theta & -\sin \theta \\
\sin \theta & \cos \theta
\end{array}\right]
$$

es una matriz de rotación. Los vórtices ópticos son un ejemplo muy utilizado en laboratorios de este tipo de haces. Otro ejemplo es un haz rotacionalmente invariante $\Gamma\left(\mathbf{r}_{1}, \mathbf{r}_{2}\right)=\Gamma\left(r_{1}, r_{2}\right)$.

La simetría de rotación también se cumple en la WD y en la AF de los haces rotacionalmente simétricos:

$$
\begin{gathered}
W(\mathbf{r}, \mathbf{p})=W[\mathbf{M}(\theta) \mathbf{r}, \mathbf{M}(\theta) \mathbf{p}], \\
A(\mathbf{r}, \mathbf{p})=A[\mathbf{M}(\theta) \mathbf{r}, \mathbf{M}(\theta) \mathbf{p}] .
\end{gathered}
$$

Esto permite simplificar el método de PST para este tipo de haces de forma significativa. En concreto, la tesis [11] propone un método que permite recuperar la AF del haz, y por tanto su MI, a partir de la medida de un conjunto de proyecciones asociadas con la FRFT antisimétrica para ángulo cubriendo un rango $\pi / 2$ [16].

Consideremos la AF en tres sistemas de coordenadas diferentes:

1. En coordenadas cartesianas, denotada por $A(\mathbf{r}, \mathbf{p})$.

2. En coordenadas polares mezclando los planos $x y$ y $u v$, denotada por $\tilde{\mathrm{A}}\left(r, \rho_{r}, p, \rho_{p}\right)$.

3. En coordenadas polares mezclando los planos $x u$ e $y v$, denotada por $\hat{\mathrm{A}}\left(R_{x}, \alpha_{x}, R_{y}, \alpha_{y}\right)$.

Uno puede cambiar entre los diferentes sistemas de coordenadas utilizando las siguientes identidades:

$$
\begin{array}{ll}
x=r \cos \theta_{r}=R_{x} \cos \alpha_{x}, & y=r \sin \theta_{r}=R_{y} \cos \alpha_{y}, \\
u=r \cos \theta_{p}=R_{x} \sin \alpha_{x}, & v=p \sin \theta_{p}=R_{y} \sin \alpha_{y} .
\end{array}
$$

La pieza clave para la simplificación de la PST es la relación entre un espectro fraccionario de potencia del haz y una sección de la AF del haz en coordenadas polares en los planos $x u$ e yv:

$$
\hat{\mathrm{A}}\left(R_{x}, \alpha_{x}, R_{y}, \alpha_{y}\right)=\frac{1}{\sigma^{2}} \int \mathrm{d} \mathbf{r}^{\prime} \mathrm{S}^{\gamma_{x}}, \gamma_{y}\left(\mathbf{r}^{\prime}\right) \exp \left(-\mathrm{i} 2 \pi \frac{x^{\prime} R_{x}+y^{\prime} R_{y}}{\sigma^{2}}\right) .
$$

donde $\alpha_{i}=\pi / 2-\gamma_{i}$. Utilizando Ec. (21) se puede comprobar que únicamente es necesario medir la proyecciones asociadas a la FRFT antisimétrica para un ángulo cubriendo un rango $\pi / 2$ para recuperar toda la información de la MI del haz [16].

Para recuperar la MI del haz necesitamos realizar la transformada de Fourier a la AF, por lo que es importante expresar la AF en coordenadas cartesianas a partir de las secciones polares obtenidas usando los espectros fraccionarios de potencia, Ec. (21). Para ello considere la relación entre la sección polar obtenida a partir de la proyección antisimétrica asociada con ángulo gamma:

$$
\hat{\mathrm{A}}\left(R \cos \theta_{R}, \frac{\pi}{2}-\gamma, R \sin \theta_{R}, \frac{\pi}{2}+\gamma\right)=\tilde{\mathrm{A}}\left(r_{0}, \theta_{r_{0}}, R|\cos \gamma|, 2 \theta_{R}+\theta_{r_{0}}\right) .
$$

A la izquierda tenemos la información obtenida al aplicar la transformada de Fourier a una proyección antisimétrica para ángulo $\gamma$, lo que equivale en coordenadas $\tilde{A}$ a un círculo de radio $R|\cos \gamma|$ para variables $r, \theta$ fijas. Por lo tanto, toda la información del haz se puede obtener aplicando el siguiente método:

1. Medir espectros fraccionarios de Fourier antisimétricos para ángulo $\gamma$ variando en un rango $\pi / 2$.

2. Aplicar la transformada de Fourier a cada espectro fraccionario de Fourier para obtener $\hat{A}$.

3. Por cada sección de Â, extraer un círculo de la sección en coordenadas Ã.

4. Interpolar valores entre círculos de Ã para obtener una sección de Ã con variación de valores suave.

5. Aplicar transformada de Fourier inversa para obtener la MI.

Utilizando dicho método simplificado se comprobó mediante simulación numérica que la técnica produce resultados correctos en un tiempo razonable. En la Fig. 5 se muestran dos secciones de $\tilde{A}$ para un haz parcialmente coherente con MI:

$$
\Gamma_{\Sigma}\left(\mathbf{r}_{1}, \mathbf{r}_{2}\right)=\frac{1}{2}\left\{L_{2}^{2}\left(\mathbf{r}_{1}\right)\left[L_{2}^{2}\left(\mathbf{r}_{2}\right)\right]^{*}+L_{2}^{-2}\left(\mathbf{r}_{1}\right)\left[L_{2}^{-2}\left(\mathbf{r}_{2}\right)\right]^{*}\right\}
$$


siendo $L_{p}^{l}$ la amplitud de campo asociada a un haz Laguerre-Gauss. Los resultados son muy similares con la teoría, lo que demuestra la validez de la técnica.

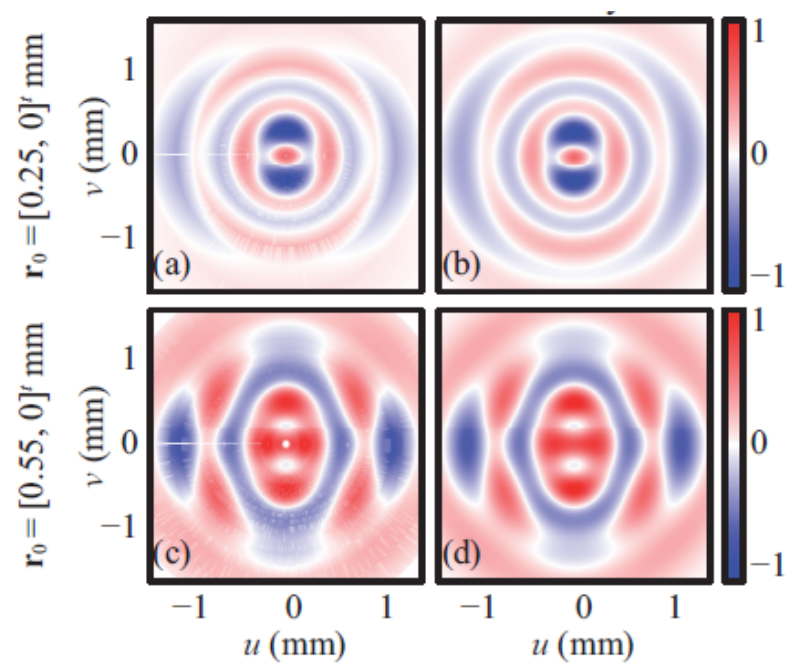

Fig. 5. Secciones de Ã obtenidas para el haz $\Gamma_{\Sigma}$ mediante simulación numérica.

Además, la técnica se comprobó experimentalmente utilizando un haz coherente colimado atravesando una apertura circular con chirp. Para la obtención de los espectros fraccionarios de potencia antisimétricos se utilizó el mismo sistema que el propuesto para haces separables en coordenadas cartesianas, refiérase a Fig. 3.

Este método ha demostrado ser útil para la caracterización de haces con simetría rotacional. El sistema óptico necesario está formado por elementos analógicos fácilmente encontrados en laboratorios de óptica.

\section{Coherenscopía}

Las técnicas propuestas hasta ahora han sido optimizadas para caracterizar haces con ciertas simetrías. El método que se explica en esta Sección, llamado coherenscopía, es eficiente para analizar la información de coherencia de haces generales, es decir, sin ninguna simetría aparente.

La base de la coherenscopía es encontrar un conjunto de rotaciones en el espacio de fases que [17]:

1. Cubra todas las direcciones necesarias para caracterizar completamente el haz,

2. Sea óptimo para la recuperación de la información de coherencia del haz (MI).

El resultado es un conjunto de rotaciones que incluye una rotación para ángulo $\alpha$ en el espacio real, plano $x y$ y $u v$, acompañado por una rotación para ángulo $\gamma$ en el plano $y v$ (parte vertical de la FRFT). A partir de las proyecciones asociadas a dichas rotaciones, $P^{\alpha, \gamma}$, podemos recuperar la MI del haz para un punto de referencia $\mathbf{r}_{0}=\left(x_{0}, y_{0}\right)$ comparado con el resto de puntos de una línea que pasa por el punto $\mathbf{r}_{0}$ con dirección $\mathbf{n}_{\alpha}=(-\sin \alpha, \cos \alpha)$ :

$$
\Gamma\left(\mathbf{r}_{0}, \boldsymbol{r}_{0}+s \mathbf{n}_{\alpha}\right)=\int \mathrm{d} v W_{x_{0}, \alpha}\left(\frac{2 y_{0}+s \cos \alpha}{2}, v\right) \exp [\mathrm{i} 2 \pi v(s \cos \alpha)],
$$

donde $s$ es el parámetro que define el punto del segmento para el que calculamos la MI relativa al punto de referencia $\mathbf{r}_{0}$ y $W_{x_{0}, \alpha}$ es la WD que se puede obtener a partir del conjunto de proyecciones $P^{\alpha, \gamma}$ para $\alpha$ constante y $\gamma$ variable.

Siguiendo este método se puede recuperar la MI entre cualesquiera dos puntos del haz en una línea, con la ventaja de que únicamente se necesita procesar un conjunto limitado de proyecciones para obtener parte de la información de la MI. Es decir, no se tiene que obtener la WD del haz completo para recuperar la información de coherencia del haz entre dichos puntos.

Para medir las proyecciones $P^{\alpha, \gamma}$ se usó en la tesis el sistema óptico propuesto en [18] compuesto por dos SLMs que implementan una máscara de fase con la siguiente función de modulación:

$$
L_{j}^{\alpha, \gamma}(\mathbf{r})=\exp \left[-\frac{\mathrm{i} \pi}{\lambda g_{j}}(x \cos \alpha-y \sin \alpha)^{2}\right] \exp \left[-\frac{\mathrm{i} \pi}{\lambda \mathrm{f}_{j}(\gamma)}(x \sin \alpha+y \cos \alpha)^{2}\right],
$$


donde $j$ es el índice que identifica a cada SLM, y

$$
\begin{gathered}
g_{1}=z, \quad \mathrm{f}_{1}(\gamma)=\frac{2 z}{2-\cot \gamma / 2}, \\
g_{2}=z / 2, \quad \mathrm{f}_{2}(\gamma)=\frac{z}{2-2 \sin \gamma} .
\end{gathered}
$$

Con el fin de demostrar el correcto funcionamiento de la técnica, en la tesis [11] se comparan los resultados experimentales de esta técnica con otra técnica completamente establecida que necesita haces coherentes. Esto permite asegurar que el método produce resultados compatibles con los resultados obtenidos por otro método bien considerado en la literatura.

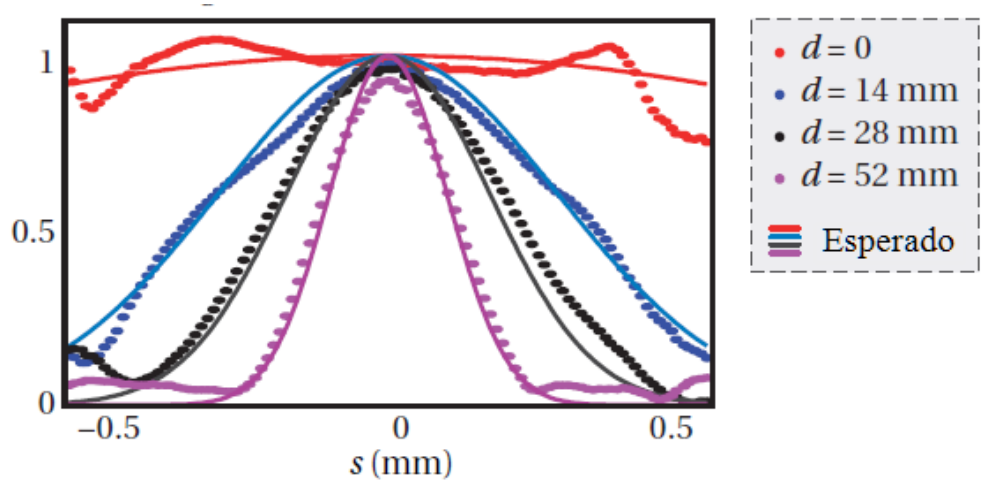

Fig. 6. Módulo del grado de coherencia para diferentes separaciones del difusor rotatorio del plano focal del sistema 4-f.

En la tesis [11] también se demuestra experimentalmente el funcionamiento de la técnica al caracterizar un haz Gauss-Schell model (GSM). El haz GSM se genera haciendo pasar el haz láser por un difusor rotante colocado en el plano focal de un sistema 4-f. Esto lo vuelve completamente incoherente. Seguidamente, el haz pasa por un SLM que codifica la intensidad y fase del haz. Desplazando el difusor a lo largo del eje óptico del sistema se controla la anchura de la gaussiana de coherencia del haz generado, siendo mayor la coherencia cuanto más cerca del plano focal del sistema 4-f se encuentre el difusor.

En la Fig. 6 se muestra el módulo del grado de coherencia del haz para el perfil de MI asociado a $\alpha=0$ usando el punto de referencia $\mathbf{r}_{0}=(0.6,0) \mathrm{mm}$ para diferentes separaciones del difusor rotatorio del plano focal del sistema 4 -f, $d$.

Por último, en la tesis se comprobó el funcionamiento del método para haces con grado de coherencia nohomogénea, que es el caso más general. Se utilizó el haz construido como superposición de haces coherentes ortogonales con MI:

$$
\Gamma\left(\mathbf{r}_{1}, \mathbf{r}_{2}\right)=\frac{1}{2}\left[\Gamma_{L_{0}^{3}}\left(\mathbf{r}_{1}, \mathbf{r}_{2}\right)+\Gamma_{L_{4}^{1}}\left(\mathbf{r}_{1}, \mathbf{r}_{2}\right)\right],
$$

donde $\Gamma_{L_{p}^{l}}$ es la MI asociada con el modo coherente de Laguerre-Gauss con índices $p$ y $l$.

Esta técnica ha demostrado ser útil para la obtención de la información de coherencia de haces parcialmente coherentes en un tiempo compatible con aplicaciones del mundo real. La información se produce de forma incremental, permitiendo el procesado en paralelo de las proyecciones obtenidas.

\section{Conclusiones}

Este trabajo ha presentado cuatro técnicas para caracterizar el estado de coherencia de haces parcialmente coherentes. Tres de las técnicas explotan simetrías en el modelo espacial del haz, mientras que la cuarta técnica permite caracterizar haces con estructura espacial desconocida. El establecimiento de estos métodos, que son fácilmente reproducibles en laboratorios de óptica, abre la posibilidad de utilizar la información local de coherencia de un haz en aplicaciones experimentales.

\section{Agradecimientos:}

Se reconoce al Ministerio de Economía y Competitividad por la financiación a través del proyecto TEC201123629 y a la Comunidad Autónoma de Madrid y el European Social Fund por el contrato de investigador de apoyo. Se agradece enormemente a Tatiana Alieva Krasheninnikova, María Luisa Calvo Padilla y José Augusto Rodrigo Martín Romo por su ayuda en la tesis, sin la cual hoy no se podría leer este artículo. 